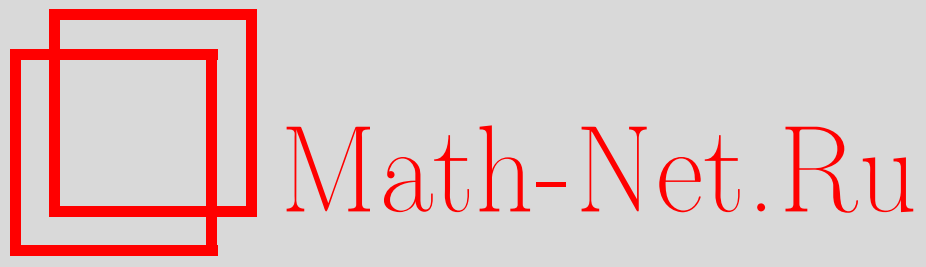

Р. О. Гринив, А. А. Шкаликов, Экспоненциальное убывание энергии решений уравнений, отвечающих некоторым операторным моделям механики, Функи. анализ и его прил., 2004, том 38, выпуск 3, 3-14

DOI: https://doi.org/10.4213/faa113

Использование Общероссийского математического портала MathNet.Ru подразумевает, что вы прочитали и согласны с пользовательским соглашением

http://www.mathnet.ru/rus/agreement

Параметры загрузки:

IP: 54.162 .127 .20

26 апреля 2023 г., 16:10:00

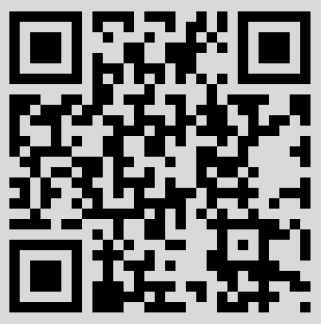


Функииональный анализ и его приложения

2004, т. 38, вып. 3, с. 3-14

УДК 517.43

\title{
Экспоненциальное убывание энергии решений уравнений, отвечающих некоторым операторным моделям механики*
}

\author{
(c) 2004. Р. О. Гринив, А. А. ШкАликов
}

Дорогому Виктору Борисовичу Лидскому
с искренним уважением и благодарностьюо

Линеаризованные уравнения движения механических систем часто записывают в операторной форме

$$
\ddot{x}+B \dot{x}+A x=0 .
$$

Здесь $x(t)$ - функция со значениями в подходящем гильбертовом пространстве $\mathscr{H}$, а $A$ и $B$-операторы в $\mathscr{H}$. В системе с бесконечным числом степеней свободы эти операторы обычно неограничены. При этом оператор $A$ отвечает за потенциальную энергию системы, является самосопряженным и равномерно положительным: $A=A^{*} \gg 0$. Оператор $B$ отвечает за гироскопические силы и силы демпфирования, что выражается в свойстве аккретивности: $\operatorname{Re}(B x, x) \geqslant 0$ при $x \in \mathscr{D}(B)$ - области определения оператора $B$.

Энергия решения $x(t)$ уравнения (1) определяется функционалом

$$
E(x(t))=\frac{1}{2}\left(\|\dot{x}\|^{2}+\left\|A^{1 / 2} x\right\|^{2}\right) .
$$

Из уравнения (1) получаем соотношение $\frac{d}{d t} E(x(t))=-\operatorname{Re}(B \dot{x}(t), \dot{x}(t)) \leqslant 0$, которое выражает диссипацию энергии во времени. Для многих механических систем наблюдается экспоненциальное убывание энергии. В случае систем с конечным числом степеней свободы (т.е. когда $A$ и $B$ - матрицы) устойчивость и экспоненциальная устойчивость изучались еще в книге Кельвина и Тейта [1]; строгие доказательства, основанные на теории Ляпунова, можно найти в книге Четаева [2]. В бесконечномерном случае задаче о нахождении условий на операторные коэффициенты уравнения (1), из которых следует экспоненциальная устойчивость, в литературе уделено много внимания; мы укажем работы [3-10], в которых можно найти дополнительные ссылки.

Далее всюду предполагаем, что $A$-самосопряженный и равномерно положительный оператор. Обозначим через $\mathscr{H}_{\theta}, \theta \in \mathbb{R}$, шкалу гильбертовых пространств, порожденных оператором $A$. При $\theta \geqslant 0$ пространство $\mathscr{H}_{\theta}$ совпадает с $\mathscr{D}\left(A^{\theta / 2}\right)$ и наделено нормой $\|x\|_{\theta}:=\left\|A^{\theta / 2} x\right\|$, а при $\theta<0$ оно определяется как пополнение пространства $\mathscr{H}$ по этой норме. Уравнение (1) можно переписать

* Работа Р. О. Гринива частично поддержана фондом Александра фон Гумбольдта в Германии. Работа А. А. Шкаликова частично поддержана грантом РФФИ №04-01-00712 и грантом Фонда поддержки ведущих научных школ НШ-1927.2003.1. 
как систему первого порядка

$$
\dot{\mathbf{x}}(t)=\mathscr{T}^{0} \mathbf{x}(t), \quad \mathbf{x}:=\left(\begin{array}{l}
x_{1}(t) \\
x_{2}(t)
\end{array}\right),
$$

где $\mathscr{T}^{0}$ - оператор в энергетическом пространстве $\mathscr{E}=\mathscr{H}_{1} \times \mathscr{H}$, определяемый равенствами

$$
\mathscr{T}^{0}=\left(\begin{array}{cc}
0 & I \\
-A & -B
\end{array}\right), \quad \mathscr{D}\left(\mathscr{T}^{0}\right)=\mathscr{H}_{2} \times\left(\mathscr{D}(B) \cap \mathscr{H}_{1}\right) .
$$

Легко видеть, что $\mathbf{x}(t)$ является решением уравнения (2) тогда и только тогда, когда $x_{1}(t)$ есть решение уравнения $(1)$, а $x_{2}(t)=\dot{x}_{1}(t)$; при этом $\|\mathbf{x}\|_{\mathscr{E}}^{2}=$ $2 E\left(x_{1}\right)$.

Имеем $\operatorname{Re}\left(\mathscr{T}^{0} x, x\right)_{\mathscr{E}}=-\operatorname{Re}\left(B x_{2}, x_{2}\right) \leqslant 0 ;$ поэтому оператор $\mathscr{T}^{0}$ диссипативен в пространстве $\mathscr{E}$ (здесь и далее мы используем терминологию книги Като [11]), а следовательно, допускает замыкание в этом пространстве. Ниже мы укажем условие, при котором замыкание $\mathscr{T}$ оператора $\mathscr{T}^{0}$ оказывается максимальным диссипативным оператором. При выполнении этого условия $\mathscr{T}$ является генератором полугруппы сжатий $\mathscr{U}(t):=\exp (t \mathscr{T})$, а решение уравнения $(2)$ определяется равенством $\mathbf{x}(t)=\mathscr{U}(t) \mathbf{x}_{0}$ при некотором $\mathbf{x}_{0} \in \mathscr{E}$. Следовательно, $E(x)$ убывает экспоненциально для любого решения $x(t)$ уравнения $(1)$ в том и только том случае, когда полугруппа $\mathscr{U}$ экспоненциально устойчива, т. е. когда тип экспоненциального роста (или убывания)

$$
\omega(\mathscr{T}):=\lim _{t \rightarrow \infty} \sup \frac{1}{t} \ln \|\mathscr{U}(t)\|
$$

полугруппы $\mathscr{U}$ отрицателен (см. [12-14]).

В работах $[3,4,6-8]$ было доказано, что $\omega(\mathscr{T})<0$, если $B$ - симметрический (или секториальный) оператор и в некотором смысле сильнее $A^{1 / 2}$. Тогда полугруппа $\mathscr{U}$ в действительности оказывается аналитической, и экспоненциальный тип $\omega(\mathscr{T})$ совпадает со спектральной абсциссой

$$
s(\mathscr{T}):=\sup \{\operatorname{Re} \lambda \mid \lambda \in \sigma(\mathscr{T})\},
$$

где $\sigma(\mathscr{T})$ обозначает спектр оператора $\mathscr{T}$ (заметим, что всегда $\omega(\mathscr{T}) \geqslant s(\mathscr{T})$ ). Равенство $\omega(\mathscr{T})=s(\mathscr{T})$ остается справедливым, если $B$ симметричен и сравним с $A^{\alpha}$ при $\alpha \in(0,1 / 2)$. Тогда [5] полугруппа $\mathscr{U}$ принадлежит классу Жеврея с показателем $1 /(2 \alpha)$. Случай, когда $B$ равномерно положителен и $A$-ограничен в смысле квадратичных форм, изучался в работе [9]; там же была получена точная верхняя граница для $\omega(\mathscr{T})$. В похожей ситуации экспоненциальная устойчивость полугруппы $\mathscr{U}$ изучалась в [10]. Отметим, что методы $[9,10]$ существенно используют симметричность оператора $B$.

Если уравнение (1) отвечает задаче механики, в которой учитываются не только силы демпфирования, но и гироскопические силы, то условие положительности оператора $B$ заменяется условием его аккретивности. Цель настоящей статьи - найти минимальные условия на аккретивный оператор $B$, которые гарантируют экспоненциальную устойчивость полугруппы $\mathscr{U}$, а стало быть, и экспоненциальное убывание энергии решений уравнения (1). Эти условия существенно менее ограничительны по сравнению с предыдущими работами и ожидаемы с механической точки зрения, но доказательство требует серьезной работы. Сформулируем основной результат. 
Теорема 1. Пусть $B$ - секториалъный и равномерно аккретивный оператор, причем линеал $\mathscr{D}(B) \cap \mathscr{H}_{1}$ плотен в $\mathscr{H}_{1}$. Если $B$, рассматриваемый как оператор из $\mathscr{H}_{1}$ в $\mathscr{H}_{-1}$, ограничен, т.е. если найдется постоянная $c>0$, такая, что

$$
|(B x, x)| \leqslant c\left|\left(A^{1 / 2} x, A^{1 / 2} x\right)\right| \quad \forall x \in \mathscr{D}(B) \cap \mathscr{H}_{1},
$$

то определенный равенством (3) оператор $\mathscr{T}^{0}$ имеет $m$-диссипативное замыкание $\mathscr{T}$, а полугруппа $\mathscr{U}(t)=\exp (t \mathscr{T})$ экспоненциально устойчива.

Попутно мы установим критерий максимальной диссипативности замыкания оператора $\mathscr{T}^{0}$. Отметим, что оценка (4) является не только достаточной, но и необходимой для экспоненциальной устойчивости полугруппы $\mathscr{U}$ (см. лемму 8). Условия секториальности и равномерной аккретивности оператора $B$ необходимыми для экспоненциальной устойчивости не являются, но они «очень близки» к необходимым. В частности, несложно показать, что эти условия необходимы, если $B$ коммутирует с $A$ в том смысле, что $B$ есть функция $A$. Например, если $B=I+i A$ (нет секториальности) или $B=A^{-1}$ (нет равномерной аккретивности), то экспоненциальной устойчивости не будет.

С различными конкретными задачами из теории упругости и гидродинамики, которые приводят к уравнению (1) с неограниченными операторами $A$ и $B$, можно познакомиться, например, в статьях $[15,16]$.

\section{§1. Предварительные сведения}

1.1. Во всей работе используются стандартные обозначения: $\mathscr{D}(T)$ - область определения оператора $T$, действующего в пространстве $\mathscr{H}, \sigma(T)$ и $\rho(T)-$ его спектр и резольвентное множество. Числовой образ $\Theta(T)$ определяется как множество в комплексной плоскости $\mathbb{C}$ равенством

$$
\Theta(T)=\{(T u, u) \mid u \in \mathscr{D}(T),\|u\|=1\} .
$$

Оператор $T$ называется аккретивным (диссипативным), если $\Theta(T)$ принадлежит правой (левой) полуплоскости; $T$ называется секториальным с полууглом $\varphi \in(0, \pi / 2)$, если $\Theta(T)$ лежит в секторе $|\arg z| \leqslant \varphi, z \in \mathbb{C} ; T$ называется равномерно аккретивным, если $T-\delta$ является аккретивным при некотором $\delta>0$. Аккретивный оператор называется $m$-аккретивным (максимально аккретивным), если левая полуплоскость принадлежит его резольвентному множеству. Аналогично определяются $m$-диссипативные и $m$-секториальные операторы. Известно $[11$, гл. $\mathrm{V}, \S 3.10]$, что аккретивный, диссипативный или секториальный оператор является $m$-аккретивным, $m$-диссипативным или $m$-секториальным в том и только том случае, когда он не допускает собственных аккретивных, диссипативных или секториальных расширений соответственно.

Если $T$ - секториальный оператор, то квадратичная форма

$$
\mathfrak{t}_{R}[u]=\operatorname{Re}(T u, u), \quad u \in \mathscr{D}(T),
$$

неотрицательна и замыкаема [11, теорема VI.2.9]. Согласно теоремам о представлении [11, теоремы VI.2.1 и VI.2.23], существует неотрицательный самосопряженный оператор $T_{R}$ (называемый действительной частью оператора $T$ ), 
такой, что $\mathscr{D}(T) \subset \mathscr{D}\left(T_{R}^{1 / 2}\right)$ и $\mathfrak{t}_{R}[u]=\left(T_{R} u, u\right)$ для всех $u \in \mathscr{D}\left(T_{R}\right)$. Если $\varphi-$ полуугол оператора $T$, то существует ограниченный самосопряженный оператор $S,\|S\| \leqslant \operatorname{tg} \varphi$, такой, что

$$
T \subset T_{R}^{1 / 2}(I+i S) T_{R}^{1 / 2},
$$

причем $T=T_{R}^{1 / 2}(I+i S) T_{R}^{1 / 2}$ в том и только том случае, когда $T m$-диссипативен (см. [11, теорема VI.3.2]).

Определенные выше понятия сохраняют смысл и для операторов, действующих из $\mathscr{H}_{1}$ в $\mathscr{H}_{-1}$. В частности, $T: \mathscr{H}_{1} \rightarrow \mathscr{H}_{-1}$ называется аккретивным, если $(T x, x) \geqslant 0$ для всех $x \in \mathscr{D}(T) \subset \mathscr{H}_{1}$, а $(, \quad)$ - дуальное скалярное произведение в $\mathscr{H}_{-1} \times \mathscr{H}_{1}$. (Здесь мы используем одно и то же обозначение и для дуального, и для обычного скалярных произведений.) Оператор $T m$-аккретивен, если выполнено одно из следующих эквивалентных условий:

(i) $T$ не имеет собственных аккретивных расширений;

(ii) при некотором (а тогда при любом) $\lambda$ из открытой правой полуплоскости $\mathbb{C}^{+}$образ $\mathscr{R}(T-\lambda)$ совпадает с $\mathscr{H}_{-1} ;$

(iii) сопряженный оператор $T^{*}: \mathscr{H}_{1} \rightarrow \mathscr{H}_{-1}$ аккретивен;

(iv) оператор $A^{-1 / 2} B A^{-1 / 2} m$-аккретивен в $\mathscr{H}$.

1.2. Экспоненциальная устойчивость. Имеется обширная литература о необходимых и достаточных условиях на операторы в банаховых пространствах для того, чтобы они генерировали равномерно ограниченные или экспоненциально устойчивые полугруппы (см., например, [12-14, 17-24] и имеющиеся там ссылки). Нам будет удобно использовать результат Герхарта [25] для гильбертова пространства. Напомним, что пространство Харди $H^{\infty}(\alpha)$ в полуплоскости $\Pi^{+}(\alpha)=\{z \in \mathbb{C} \mid \operatorname{Re} z>\alpha\}$ состоит из голоморфных и равномерно ограниченных в $\Pi^{+}(\alpha)$ функций. В частности, резольвента $(T-\lambda)^{-1}$ оператора $T$ в гильбертовом пространстве $\mathscr{H}$ принадлежит $H^{\infty}(\alpha)$ в том и только том случае, когда $\Pi^{+}(\alpha) \subset \rho(T)$ и значения $\left\|(T-\lambda)^{-1}\right\|$ равномерно ограничены в $\Pi^{+}(\alpha)$.

ТЕОРема ГерХАРТА [25]. Экспоненииалъный тип $C_{0}$-полугруппы, генерируемой оператором $T$ в гильбертовом пространстве $\mathscr{H}$, определяется равенством

$$
\omega(T)=\inf \left\{\alpha \in \mathbb{R} \mid(T-\lambda)^{-1} \in H^{\infty}(\alpha)\right\} .
$$

Из этой теоремы получается такое известное утверждение (см. [14, Theоrem V.1.11]).

СлЕДСтвИЕ 2. Если Т есть $m$-диссипативный оператор, то генерируемая им полугруппа сжатий является экспоненциалъно устойчивой тогда и только тогда, когда

$$
\sup _{\lambda \in \mathbb{C}^{+}}\left\|(T-\lambda)^{-1}\right\|<\infty .
$$

Здесъ $\mathbb{C}^{+}$-открытая правая полуплоскость.

\section{§2. Оператор $\mathscr{T}^{0}$ и его свойства}

Наша ближайшая цель - описать замыкание $\mathscr{T}$ оператора $\mathscr{T}^{0}$, определенного равенством (3), и выяснить, когда $\mathscr{T}$ является $m$-диссипативным. Всюду далее предполагаем, что линеал $\mathscr{D}(B) \cap \mathscr{H}_{1}$ плотен в $\mathscr{H}_{1}$. 
Заметим, что $\|A x\|_{-1}=\|x\|_{1}$ при $x \in \mathscr{H}_{2}$, согласно определению шкалы пространств $\mathscr{H}_{\theta}$; следовательно, оператор $A$ продолжается по непрерывности как унитарный оператор $\widetilde{A}$ из $\mathscr{H}_{1}$ в $\mathscr{H}_{-1}$. Далее, если $\operatorname{Re}(B x, x) \geqslant 0$ при $x \in$ $\mathscr{D}(B) \cap \mathscr{H}_{1}$, то $B$ замыкаем как оператор из $\mathscr{H}_{1}$ в $\mathscr{H}_{-1}$, а его замыкание $\widetilde{B}$ удовлетворяет неравенству $\operatorname{Re}(\widetilde{B} x, x) \geqslant 0$ при $x \in \mathscr{D}(\widetilde{B}) \subset \mathscr{H}_{1}$, где ( , )дуальное скалярное произведение в $\mathscr{H}_{-1} \times \mathscr{H}_{1}$ (см. [11, теорема V.3.4]).

Лемма 3. Если В допускает замъкание $\widetilde{B}$ как оператор из $\mathscr{H}_{1}$ в $\mathscr{H}_{-1}$, то $\mathscr{T}^{0}$ допускает замыкание $\mathscr{T}$, которое определено равенствами

$$
\begin{gathered}
\mathscr{T}\left(\begin{array}{l}
x_{1} \\
x_{2}
\end{array}\right)=\left(\begin{array}{c}
x_{2} \\
-\widetilde{A} x_{1}-\widetilde{B} x_{2}
\end{array}\right), \\
\mathscr{D}(\mathscr{T})=\left\{\left(\begin{array}{l}
x_{1} \\
x_{2}
\end{array}\right) \mid x_{1} \in \mathscr{H}_{1}, x_{2} \in \mathscr{D}(\widetilde{B}), \widetilde{A} x_{1}+\widetilde{B} x_{2} \in \mathscr{H}\right\} .
\end{gathered}
$$

В частности, если $B$ аккретивен, то $\mathscr{T}_{0}$ замыкаем, и его замыкание $\mathscr{T}$ диссипативный оператор.

ДокАЗАТЕЛьство. Мы докажем сначала, что оператор $\mathscr{T}$, определенный равенствами (6), замкнут. Так как $\mathscr{T} \supset \mathscr{T}^{0}$, то, тем самым, оператор $\mathscr{T}^{0}$ допускает замыкание. Обозначим это замыкание через $\widetilde{\mathscr{T}} ;$ тогда равенство $\widetilde{\mathscr{T}}=\mathscr{T}$ будет следовать из включения $\mathscr{T} \subset \widetilde{\mathscr{T}}$.

Покажем, что $\mathscr{T}$ замкнут. Пусть $\mathbf{x}^{n}=\left(x_{1}^{n}, x_{2}^{n}\right)^{T} \in \mathscr{D}(\mathscr{T})$ и $\mathbf{x}^{n} \rightarrow \mathbf{x}=$ $\left(x_{1}, x_{2}\right)^{T}, \mathscr{T} \mathbf{x}^{n} \rightarrow \mathbf{y}=\left(y_{1}, y_{2}\right)^{T}$ в пространстве $\mathscr{E}$ при $n \rightarrow \infty$. Тогда $x_{1}^{n} \rightarrow x_{1}$, $x_{2}^{n} \rightarrow x_{2}=y_{1}$ в $\mathscr{H}_{1}$ и $-\widetilde{A} x_{1}^{n}-\widetilde{B} x_{2}^{n} \rightarrow y_{2}$ в $\mathscr{H}$. Следовательно, $x_{1} \in \mathscr{H}_{1}$, $\widetilde{A} x_{1}^{n} \rightarrow \widetilde{A} x_{1},-\widetilde{B} x_{2}^{n} \rightarrow \widetilde{A} x_{1}+y_{2}$ в $\mathscr{H}_{-1}$. В силу замкнутости $\widetilde{B}$ имеем $x_{2} \in \mathscr{D}(\widetilde{B})$ и $-\widetilde{B} x_{2}=\widetilde{A} x_{1}+y_{2}$. Таким образом, $\mathbf{x} \in \mathscr{D}(\mathscr{T})$ и $\mathscr{T} \mathbf{x}=\mathbf{y}$, т. е. $\mathscr{T}$ замкнут.

Остается обосновать включение $\mathscr{T} \subset \widetilde{\mathscr{T}}$. Для этого зафиксируем произвольный элемент $\mathbf{x}=\left(x_{1}, x_{2}\right)^{T} \in \mathscr{D}(\mathscr{T})$ и найдем последовательность элементов $\mathbf{x}^{n} \in \mathscr{D}\left(\mathscr{T}^{0}\right)$, такую, что $\mathbf{x}^{n} \rightarrow \mathbf{x}, \mathscr{T}^{0} \mathbf{x}^{n} \rightarrow \mathscr{T} \mathbf{x}$ в пространстве $\mathscr{E}$ при $n \rightarrow \infty$. Пусть $\mathbf{y}=\left(y_{1}, y_{2}\right)^{T}=\mathscr{T} \mathbf{x}$. Так как $x_{2} \in \mathscr{D}(\widetilde{B})$, то существует последовательность элементов $x_{2}^{n} \in \mathscr{D}(B) \cap \mathscr{H}_{1}$, такая, что $x_{2}^{n} \rightarrow x_{2}$ в $\mathscr{H}_{1}$ и $B x_{2}^{n} \rightarrow \widetilde{B} x_{2}$ в $\mathscr{H}_{-1}$ при $n \rightarrow \infty$. Положим $x_{1}^{n}:=-A^{-1}\left(y_{2}+B x_{2}^{n}\right), \mathbf{x}^{n}:=\left(x_{1}^{n}, x_{2}^{n}\right)^{T}$ и заметим, что $\mathbf{x}^{n} \in \mathscr{D}\left(\mathscr{T}^{0}\right)$. Далее, имеем $x_{2}^{n} \rightarrow x_{2}=y_{1}$ в $\mathscr{H}_{1}$. Так как $B x_{2}^{n} \rightarrow \widetilde{B} x_{2}$ в $\mathscr{H}_{-1}$, то $x_{1}^{n} \rightarrow-\tilde{A}^{-1}\left(y_{2}+\widetilde{B} x_{2}\right)=x_{1}$ в $\mathscr{H}_{1} ;$ следовательно, $\mathbf{x}^{n} \rightarrow \mathbf{x}$ в пространстве $\mathscr{E}$. Согласно определению, $A x_{1}^{n}+B x_{2}^{n}=-y_{2} ;$ поэтому $\mathscr{T}^{0} \mathbf{x}^{n}=\left(x_{2}^{n}, y_{2}\right)^{T} \rightarrow \mathbf{y}$ в $\mathscr{E}$. Таким образом, $\mathscr{T} \subset \widetilde{\mathscr{T}}$, и лемма доказана.

Tеорема 4. Oператор $\mathscr{T}$ m-диссипативен в том и толъко том случае, когда т-аккретивным является оператор $\widetilde{B}$ - замыкание $B$ как оператора из $\mathscr{H}_{1}$ в $\mathscr{H}_{-1}$.

ДокАЗАТЕЛЬСтво. Пусть $\widetilde{B} m$-аккретивен. Для диссипативного оператора $\mathscr{T}$ образ оператора $\mathscr{T}-\mathscr{I}$ (здесь $\mathscr{I}$ - тождественный оператор в $\mathscr{E}$ ) является замкнутым; поэтому достаточно доказать, что образ оператора $\mathscr{T}-\mathscr{I}$ плотен в пространстве $\mathscr{E}$. 
Предположим, что существует элемент $\mathbf{y}=\left(y_{1}, y_{2}\right)^{T} \in \mathscr{E}$, ортогональный образу оператора $\mathscr{T}-\mathscr{I}$. Тогда

$$
(\mathscr{T} \mathbf{x}, \mathbf{y})_{\mathscr{E}}-(\mathbf{x}, \mathbf{y})_{\mathscr{E}}=\left(x_{2}, y_{1}\right)_{1}-\left(x_{1}, y_{1}\right)_{1}-\left(\widetilde{A} x_{1}+\widetilde{B} x_{2}, y_{2}\right)-\left(x_{2}, y_{2}\right)=0
$$

для всех $\mathbf{x}=\left(x_{1}, x_{2}\right) \in \mathscr{D}(\mathscr{T})$. Полагая $x_{2}=0$, получаем, что равенство $\left(A x_{1}, y_{1}+y_{2}\right)=0$ выполняется при всех $x_{1} \in \mathscr{H}_{2}$, а потому $y_{2}=-y_{1}$. Теперь, полагая $x_{1}=0$, находим, что

$$
\left(x_{2}, y_{1}\right)_{1}+\left(B x_{2}, y_{1}\right)+\left(x_{2}, y_{1}\right)=\left(\widetilde{A} x_{2}+B x_{2}+x_{2}, y_{1}\right)=0
$$

при всех $x_{2} \in \mathscr{D}(B) \cap \mathscr{H}_{1}$. Это равенство остается справедливым при всех $x_{2} \in$ $\mathscr{D}(\widetilde{B})$. Действительно, если $x_{2} \in \mathscr{D}(\widetilde{B})$, то, согласно определению замыкания $\widetilde{B}$, найдется последовательность элементов $x_{2}^{n} \in \mathscr{D}(B) \cap \mathscr{H}_{1}$, такая, что $x_{2}^{n} \rightarrow x_{2}$ в $\mathscr{H}_{1}$ и $B x_{2}^{n} \rightarrow \widetilde{B} x_{2}$ в $\mathscr{H}_{-1}$. Заменяя в (7) $x_{2}$ на $x_{2}^{n}$, переходя к пределу и учитывая, что $y_{1} \in \mathscr{H}_{1}$, получаем равенство $(7)$ при $x_{2} \in \mathscr{D}(\widetilde{B})$.

Положим $\widetilde{C}=\widetilde{A}+\widetilde{B}+\tilde{I}$, где $\tilde{I}$ - оператор естественного вложения пространства $\mathscr{H}_{1}$ в $\mathscr{H}_{-1}$. Так как $\widetilde{A}+\tilde{I}$ ограничен, то $\widetilde{C}$ - замкнутый оператор с областью определения $\mathscr{D}(\widetilde{C})=\mathscr{D}(\widetilde{B})$, причем $\widetilde{C}^{*}=\widetilde{A}+\widetilde{B}^{*}+\tilde{I}$. Из равенства $(7)$, которое по непрерывности справедливо при всех $x_{2} \in \mathscr{D}(\widetilde{B})$, следует, что $y_{1} \in \operatorname{ker} \widetilde{C}^{*}$.

Но если $\widetilde{B}$ является $m$-аккретивным, то $\widetilde{B}^{*}$ аккретивен, а потому

$$
0=\operatorname{Re}\left(\widetilde{C}^{*} y_{1}, y_{1}\right) \geqslant\left(\widetilde{A} y_{1}, y_{1}\right)+\left\|y_{1}\right\|^{2} .
$$

Следовательно, $y_{1}=-y_{2}=0$ и $\mathbf{y}=0$. Тем самым доказано, что $\mathscr{T}$ является $m$-диссипативным.

Обратно, если $\mathscr{T} m$-диссипативен, то уравнение $(\mathscr{T}-\mathscr{I})\left(x_{1}, x_{2}\right)^{T}=\left(y_{1}, 0\right)^{T}$, эквивалентное уравнению $(\widetilde{A}+\widetilde{B}+\tilde{I}) x_{2}=\widetilde{A} y_{1}$, разрешимо при всех $y_{1} \in \mathscr{H}_{1}$. Значит, образ оператора $\widetilde{A}+\widetilde{B}+\tilde{I}$ совпадает с $\mathscr{H}_{-1}$. Если бы оператор $\widetilde{B}$ имел нетривиальное аккретивное расширение $\widehat{B}$ из $\mathscr{H}_{1}$ в $\mathscr{H}_{-1}$, то оператор $\widehat{C}:=$ $\widetilde{A}+\widehat{B}+\tilde{I}$ обладал бы нетривиальным ядром, что невозможно ввиду соотношения

$$
\operatorname{Re}(\widehat{C} x, x) \geqslant(\widetilde{A} x, x)+\|x\|^{2} .
$$

Таким образом, $\widetilde{B}$ не допускает нетривиальных аккретивных расширений из $\mathscr{H}_{1}$ в $\mathscr{H}_{-1}$, а потому является $m$-аккретивным. Теорема доказана.

ЗАмЕчАНИЕ 5. Заметим, что если $B-$ квазиаккретивный оператор, т. е. $\operatorname{Re}(B x, x) \geqslant-\alpha(x, x)$ при некотором $\alpha>0$, то (так же, как при $\alpha=0$ ) существует его замыкание $\widetilde{B}$ как оператора из $\mathscr{H}_{1}$ в $\mathscr{H}_{-1}$. В этом случае

$$
-\operatorname{Re}\left(\mathscr{T}^{0} \mathbf{x}, \mathbf{x}\right) \geqslant-\alpha\left(x_{2}, x_{2}\right) \geqslant-\alpha(\mathbf{x}, \mathbf{x}), \quad \mathbf{x}=\left(x_{1}, x_{2}\right)^{T},
$$

т. е. $\mathscr{T}^{0}$ квазидиссипативен. Теорему 4 можно переформулировать следующим образом: если $\alpha>0$, то оператор $\mathscr{T}-\alpha$ m-диссипативен ( $и$ полугруппа $\exp (t \mathscr{T})$ имеет экспоненииальный тип $\leqslant \alpha)$ в том и только том случае, когда $\widetilde{B}+\alpha m$-аккретивен. При $\alpha<0$ второе неравенство в (8) не выполняется, и из равномерной аккретивности оператора $B$ не следует равномерной диссипативности оператора $\mathscr{T}$. Это служит объяснением, почему доказательство теоремы 1 не получается на простом пути. 
Наш следующий шаг - получение представления для резольвенты оператора $\mathscr{T}$. Положим при $\lambda \in \mathbb{C}$

$$
\widetilde{L}(\lambda)=\lambda^{2} \tilde{I}+\lambda \widetilde{B}+\widetilde{A}, \quad \mathscr{D}(\widetilde{L})=\mathscr{D}(\widetilde{B}) \subset \mathscr{H}_{1},
$$

рассматривая $\widetilde{L}(\lambda)$ как оператор из $\mathscr{H}_{1}$ в $\mathscr{H}_{-1}$. В общем случае нельзя сказать, что $\widetilde{L}(\lambda)$ есть расширение Лаплас-символа $L(\lambda)=\lambda^{2}+\lambda B+A$ уравнения (1) как оператора из $\mathscr{H}_{1}$ в $\mathscr{H}_{-1}$, поскольку может случиться даже, что $\mathscr{D}(L)=\mathscr{D}(A) \cap$ $\mathscr{D}(B)=\{0\}$. Множество значений $\lambda \in \mathbb{C} \backslash\{0\}$, для которых оператор $\widetilde{L}(\lambda)$ имеет ограниченный обратный оператор из $\mathscr{H}_{-1}$ в $\mathscr{H}_{1}$, называется резольвентным множеством пучка $\widetilde{L}$, а дополнение к этому множеству в $\mathbb{C} \backslash\{0\}$ - его спектром. Отметим, что в случае $\mathscr{D}(B) \supset \mathscr{D}(A)$ оператор $L(\lambda)$ корректно определен в $\mathscr{H}$ на области $\mathscr{D}(L)=\mathscr{H}_{2}$. Но спектры пучков $L$ и $\widetilde{L}$ могут не совпадать, хотя дискретные части спектров совпадают [26].

Следующая теорема показывает, что спектры оператора $\mathscr{T}$ и пучка $\widetilde{L}(\lambda)$ тесно связаны между собой.

Теорема 6. Пусть В замыкаем как оператор из $\mathscr{H}_{1}$ в $\mathscr{H}_{-1}$, и пусть $\widetilde{B}$ обозначает это замыкание. Тогда ненулевой спектр оператора $\mathscr{T}$ совпадает со спектром $\sigma(\widetilde{L})$ пучка $\widetilde{L}$, причем для всех ненулевых $\lambda \notin \sigma(\widetilde{L})$ справедливо представление

$$
(\mathscr{T}-\lambda \mathscr{I})^{-1}=\left(\begin{array}{cc}
\lambda^{-1}\left(\widetilde{L}^{-1}(\lambda) \widetilde{A}-I\right) & -\widetilde{L}^{-1}(\lambda) \\
\widetilde{L}^{-1}(\lambda) \widetilde{A} & -\lambda \widetilde{L}^{-1}(\lambda)
\end{array}\right) .
$$

ДокАЗАТЕЛЬСтво. Пусть $0 \neq \lambda \in \rho(\mathscr{T})$; тогда при любом $\mathbf{y}=\left(y_{1}, y_{2}\right)^{T} \in \mathscr{E}$ существует единственный элемент $\mathbf{x}=\left(x_{1}, x_{2}\right)^{T} \in \mathscr{D}(\mathscr{T})$, такой, что $(\mathscr{T}-\lambda \mathscr{I}) \mathbf{x}=\mathbf{y}$. Записывая это уравнение по координатам, получаем систему

$$
\begin{gathered}
x_{2}-\lambda x_{1}=y_{1}, \\
-\widetilde{A} x_{1}-\widetilde{B} x_{2}-\lambda x_{2}=y_{2} .
\end{gathered}
$$

Подставив $x_{1}=\lambda^{-1}\left(x_{2}-y_{1}\right)$ во второе уравнение, получим $\widetilde{L}(\lambda) x_{2}=-\lambda y_{2}+\widetilde{A} y_{1}$. Если $y_{2}=0$, а $y_{1}$ пробегает все пространство $\mathscr{H}_{1}$, то $\widetilde{L}(\lambda) x_{2}$ пробегает $\mathscr{H}_{-1}$. Следовательно, образ оператора $\widetilde{L}(\lambda)$ совпадает с $\mathscr{H}_{-1}$. Если $x_{2}$ принадлежит ядру оператора $\widetilde{L}(\lambda)$, то $\mathbf{x}=\left(\lambda^{-1} x_{2}, x_{2}\right)^{T}$ принадлежит ядру оператора $\mathscr{T}-\lambda \mathscr{I}$. Поэтому ядро оператора $\widetilde{L}(\lambda)$ тривиально и $\widetilde{L}(\lambda)$, будучи замкнутым оператором, является ограниченно обратимым. Представление (9) получается теперь из выписанной системы прямым вычислением.

Обратно, пусть $0 \neq \lambda \in \rho(\widetilde{L})$. Обозначим через $\mathscr{B}_{\lambda}$ оператор, определенный в правой части формулы (9). Фиксируем произвольный элемент $\mathbf{y}=\left(y_{1}, y_{2}\right)^{T} \in \mathscr{E}$ и положим $\mathbf{x}=\left(x_{1}, x_{2}\right)^{T}:=\mathscr{B}_{\lambda} \mathbf{y}$. Так как оператор $\widetilde{L}^{-1}(\lambda)$ переводит $\mathscr{H}_{-1}$ в $\mathscr{D}(\widetilde{B}) \subset \mathscr{H}_{1}$, то $x_{1} \in \mathscr{H}_{1}, x_{2} \in \mathscr{D}(\widetilde{B})$ и $x_{2}-\lambda x_{1}=y_{1}$. Кроме того,

$$
\begin{aligned}
\widetilde{A} x_{1}+\widetilde{B} x_{2}+\lambda x_{2}=\left[\lambda^{-1} \widetilde{A}\left(\widetilde{L}^{-1}(\lambda) \widetilde{A}-\tilde{I}\right)+\widetilde{B} \widetilde{L}^{-1}(\lambda) \widetilde{A}+\lambda \widetilde{L}^{-1}(\lambda) \widetilde{A}\right] y_{1} \\
-\left[\widetilde{A} \widetilde{L}^{-1}(\lambda)+\lambda \widetilde{B} \widetilde{L}^{-1}(\lambda)+\lambda^{2} \widetilde{L}^{-1}(\lambda)\right] y_{2}=-y_{2} .
\end{aligned}
$$


Из этих соотношений следует равенство $(\mathscr{T}-\lambda \mathscr{I}) \mathscr{B} \lambda=\mathscr{I}$. Равенство $\mathscr{B}_{\lambda}(\mathscr{T}-\lambda \mathscr{I})$ $=\mathscr{I}$ проверяется аналогично; поэтому $\mathscr{B}_{\lambda}=(\mathscr{T}-\lambda \mathscr{I})^{-1}$. Теорема доказана.

Из теорем 4 и 6 получаем

СледСТвиЕ 7. Если $\widetilde{B}$ m-аккретивен, то открытая правая полуплоскость $\mathbb{C}^{+}$принадлежит резольвентному множеству пучка $\widetilde{L}(\lambda)$.

Конечно, этот результат проще получить независимо, исходя из неравенств

$$
\begin{aligned}
\|\widetilde{L}(\lambda) x\|_{-1} & \geqslant \frac{|\lambda|}{\|x\|_{1}} \operatorname{Re}\left(\lambda^{-1} \widetilde{L}(\lambda) x, x\right) \geqslant|\lambda|^{-1}(\operatorname{Re} \lambda)\|x\|_{1}, \\
\left\|\widetilde{L}^{*}(\lambda) x\right\|_{-1} & \geqslant|\lambda|^{-1}(\operatorname{Re} \lambda)\|x\|_{1},
\end{aligned}
$$

которые справедливы при $\lambda \in \mathbb{C}^{+}$.

Теперь выясним, когда нуль принадлежит спектру оператора $\mathscr{T}$.

ЛЕмма 8. Предположим, что оператор В допускает замыкание $\widetilde{B}$ как оператор из $\mathscr{H}_{1}$ в $\mathscr{H}_{-1}$. Тогда точка $\lambda=0$ принадлежит резольвентному множеству оператора $\mathscr{T}$ в том и только том случае, когда оператор $\widetilde{B}: \mathscr{H}_{1} \rightarrow \mathscr{H}_{-1}$ ограничен.

ДоКАЗАТЕЛЬСТво. Предположим, что оператор $\mathscr{T}$ ограниченно обратим. Тогда его образ совпадает с пространством $\mathscr{E}$. Из уравнения

$$
\mathscr{T}\left(\begin{array}{l}
x_{1} \\
x_{2}
\end{array}\right)=\left(\begin{array}{l}
y_{1} \\
y_{2}
\end{array}\right)
$$

следует равенство $x_{2}=y_{1}$. Поэтому из разрешимости уравнения (10) при всех у $\in \mathscr{E}$ (в частности, при всех $y_{1} \in \mathscr{H}_{1}$ ) вытекает $\mathscr{D}(\widetilde{B}) \supset \mathscr{H}_{1}$. Но $\widetilde{B}$ замкнут; поэтому в силу теоремы о замкнутом графике $\widetilde{B}$ ограничен.

Обратно, из ограниченности оператора $\widetilde{B}$ следует существование единственного решения уравнения (10): $x_{2}=y_{1} \in \mathscr{H}_{1}=\mathscr{D}(\widetilde{B}), x_{1}=A^{-1}\left(y_{2}-\widetilde{B} x_{2}\right) \in \mathscr{H}_{1}$. Лемма доказана.

\section{§3. Доказательство основной теоремы}

Базой для доказательства теоремы 1 служит следующий результат.

Лемма 9. Пусть В-аккретивный оператор, и пусть выполнена очен$\kappa a$ (4). Тогда оператор $\mathscr{T}$ генерирует экспоненциально устойчивую полугруппу в том и только том случае, когда четыре оператор-функиии

$$
\begin{array}{llrl}
F_{1}(\lambda):=\lambda^{-1} A^{1 / 2} \widetilde{L}^{-1 / 2}(\lambda) \widetilde{A}^{1 / 2}, & & F_{2}(\lambda):=A^{1 / 2} \widetilde{L}^{-1}(\lambda), \\
F_{3}(\lambda):=\widetilde{L}^{-1}(\lambda) \widetilde{A}^{1 / 2}, & & F_{4}(\lambda):=\lambda \widetilde{L}^{-1}(\lambda)
\end{array}
$$

равномерно ограничены в открытой правой полуплоскости $\mathbb{C}^{+}$в равномерной операторной топологии пространства $\mathscr{H}$. Здесь $\widetilde{A}^{1 / 2}=\widetilde{A} A^{-1 / 2}-$ расширение onepamopa $A^{1 / 2}$ как оператора из $\mathscr{H}$ в $\mathscr{H}_{-1}$.

ДокАЗАТЕЛЬСТВо. При выполнении оценки (4) оператор $\widetilde{B}$ ограничен. Поэтому $\widetilde{B}$ является $m$-аккретивным, если $B$ аккретивен. Согласно следствию 7 , все четыре функции корректно определены при $\lambda \in \mathbb{C}^{+}$. В силу теоремы 6 
ограниченность оператора $(\mathscr{T}-\lambda \mathscr{I})^{-1}$ при $\lambda \neq 0$ в пространстве $\mathscr{E}$ эквивалентна ограниченности четырех операторов $F_{j}(\lambda)$ в пространстве $\mathscr{H}$. Принадлежность точки $\lambda=0$ резольвентному множеству оператора $\mathscr{T}$ гарантируется леммой 8. Поэтому из равномерной ограниченности указанных функций в $\mathbb{C}^{+}$ следует равномерная ограниченность оператора $(\mathscr{T}-\lambda \mathscr{I})^{-1}$ в $\mathbb{C}^{+}$. Остается воспользоваться следствием 2.

Оценка функции $F_{4}(\lambda)$ получается относительно просто (это будет сделано в следующей лемме); основная трудность заключается в оценке первых трех функций. Решающую роль сыграет следующее равенство, в справедливости которого легко убедиться непосредственной проверкой:

$$
\widetilde{L}^{-1}(\lambda)-\widetilde{L}_{0}^{-1}(\lambda)=\lambda \widetilde{L}_{0}^{-1}(\lambda) \widetilde{A}^{1 / 2} \widetilde{L}^{-1}(\lambda)-\lambda \widetilde{L}_{0}^{-1}(\lambda) \widetilde{B} \widetilde{L}^{-1}(\lambda) .
$$

Здесь

$$
L_{0}(\lambda):=\lambda^{2} I+\lambda A^{1 / 2}+A,
$$

и $\widetilde{L}_{0}(\lambda)$ - расширение $L_{0}(\lambda)$ как оператора из $\mathscr{H}_{1}$ в $\mathscr{H}_{-1}$.

Лемма 10. Пусть оператор $B$ является секториальным и равномерно аккретивным, а $\widetilde{B}$ ограничен. Обозначим через $B_{R}$ действительную часть оператора $B$, а через $\beta>0$ нижнюю грань значений квадратичной формы $\left(B_{R} x, x\right)$ на единичной сфере. Тогда справедливы оченки

(a) $\sup _{\lambda \in \mathbb{C}^{+}}\left\|\lambda \widetilde{L}^{-1}(\lambda)\right\| \leqslant 1 / \beta$,

(b) $\sup _{\lambda \in \mathbb{C}^{+}}\left\|\lambda B_{R}^{1 / 2} \widetilde{L}^{-1}(\lambda)\right\| \leqslant 1 / \sqrt{\beta}$,

(c) $\sup _{\lambda \in \mathbb{C}^{+}}\left\|\lambda B_{R}^{1 / 2} \widetilde{L}^{-1}(\lambda) B_{R}^{1 / 2}\right\| \leqslant 1$.

ДоказАтЕЛЬство. Фиксируем произвольный элемент $y \in \mathscr{H}$. Если $\lambda \in \mathbb{C}^{+}$, то уравнение $\widetilde{L}(\lambda) x=y$ разрешимо при любом $y \in \mathscr{H}_{-1}$, тем более при $y \in \mathscr{H}$. Справедливы оценки

$$
\left|\left(\lambda^{-1} \widetilde{L}(\lambda) x, x\right)\right| \geqslant\left|\operatorname{Re}\left(\lambda^{-1} \widetilde{L}(\lambda) x, x\right)\right| \geqslant \operatorname{Re}(\widetilde{B} x, x) \geqslant \beta\|x\|^{2} .
$$

Следовательно, $\|\widetilde{L}(\lambda) x\| \geqslant \beta|\lambda|\|x\|$; поэтому $\left\|\lambda \widetilde{L}^{-1}(\lambda) y\right\| \leqslant 1 / \beta\|y\|$, т. е. справедлива оценка (a).

Согласно определению оператора $B_{R}$, имеем $\left\|B_{R}^{1 / 2} x\right\|^{2}=\operatorname{Re}(B x, x)$ при $x \in$ $\mathscr{D}(B) \cap \mathscr{H}_{1}$. Поскольку замыкание $\widetilde{B}$ - ограниченный оператор из $\mathscr{H}_{1}$ в $\mathscr{H}_{-1}$, а $\mathscr{D}(B) \cap \mathscr{H}_{1}$ плотно в $\mathscr{H}_{1}$, по непрерывности имеем

$$
\left\|B_{R}^{1 / 2} x\right\|^{2}=\operatorname{Re}(\widetilde{B} x, x) \quad \forall x \in \mathscr{H}_{1} .
$$

Пользуясь неравенством (13) и доказанным неравенством (а) при $y \in \mathscr{H}$ и $\lambda \in \mathbb{C}^{+}$, получаем

$$
\begin{aligned}
\frac{\|y\|^{2}}{\beta} & \geqslant\|y\|\left\|\lambda \widetilde{L}^{-1}(\lambda) y\right\| \geqslant|\lambda|\left(y, \widetilde{L}^{-1}(\lambda) y\right)|=| \lambda \mid\left(\widetilde{L}(\lambda) \widetilde{L}^{-1}(\lambda) y, \widetilde{L}^{-1}(\lambda) y\right) \\
& \geqslant|\lambda|^{2} \operatorname{Re}\left(\widetilde{B} \widetilde{L}^{-1}(\lambda) y, \widetilde{L}^{-1}(\lambda) y\right)=|\lambda|^{2}\left\|B_{R}^{1 / 2} \widetilde{L}^{-1}(\lambda) y\right\|^{2},
\end{aligned}
$$

т. е. справедлива оценка (b). 
Множество элементов $y=B_{R}^{1 / 2} x$ плотно в $\mathscr{H}$, когда $x$ пробегает $\mathscr{H}_{1}$. Воспользовавшись оценкой

$$
\left|\left(\lambda^{-1} \widetilde{L}(\lambda) x, x\right)\right| \geqslant \operatorname{Re}(\widetilde{B} x, x)=\left\|B_{R}^{1 / 2} x\right\|^{2},
$$

получим

$$
\|y\|^{2} \leqslant|\lambda|^{-1}\left|\left(B_{R}^{-1 / 2} \widetilde{L}(\lambda) B_{R}^{-1 / 2} y, y\right)\right| \leqslant|\lambda|^{-1}\left\|B_{R}^{-1 / 2} \widetilde{L}(\lambda) B_{R}^{-1 / 2} y\right\|\|y\|,
$$

откуда следует оценка (c). Лемма доказана.

Лемма 11. Функиии $F_{2}(\lambda)$ и $F_{3}(\lambda)$ равномерно ограничены в $\mathbb{C}^{+}$в равномерной операторной топологии пространства $\mathscr{H}$.

ДокАЗАтЕльство. Пусть $L_{0}(\lambda)$ определен формулой $(12)$, и пусть $\widetilde{L}_{0}(\lambda)-$ его расширение как оператора из $\mathscr{H}_{1}$ в $\mathscr{H}_{-1}$. Элементарной проверкой (см. [4]) устанавливается, что оператор-функции

$$
\begin{array}{llrl}
G_{1}(\lambda) & =A^{1 / 2} \widetilde{L}_{0}^{-1}(\lambda) \widetilde{A}^{1 / 2}, & & G_{2}(\lambda)=\lambda A^{1 / 2} \widetilde{L}_{0}^{-1}(\lambda), \\
G_{3}(\lambda)=\lambda \widetilde{L}_{0}^{-1}(\lambda) \widetilde{A}^{1 / 2}, & & G_{4}(\lambda)=\lambda^{2} \widetilde{L}_{0}^{-1}(\lambda)
\end{array}
$$

равномерно ограничены в замкнутой правой полуплоскости. (В частности, $\left\|\left(\mathscr{T}_{0}-\lambda\right)^{-1}\right\|_{\mathscr{E}} \leqslant$ const $\left|(1+\lambda)^{-1}\right|$ при $\lambda \in \mathbb{C}^{+}$, где через $\mathscr{T}_{0}$ обозначен оператор $\mathscr{T}$, в котором $B=A^{1 / 2}$; поэтому $\mathscr{T}_{0}$ генерирует аналитическую полугруппу сжатий.)

Умножив равенство (11) слева на $A^{1 / 2}$, получим

$$
A^{1 / 2} \widetilde{L}^{-1}(\lambda)=A^{1 / 2} \widetilde{L}_{0}^{-1}(\lambda)+A^{1 / 2} \widetilde{L}_{0}^{-1}(\lambda) \widetilde{A}^{1 / 2} \lambda \widetilde{L}^{-1}(\lambda)-A^{1 / 2} \widetilde{L}_{0}^{-1}(\lambda) \widetilde{B} \lambda \widetilde{L}^{-1}(\lambda) .
$$

Первые два слагаемых равномерно ограничены в $\mathbb{C}^{+}$, поскольку функции $F_{4}(\lambda)$, $G_{1}(\lambda)$ и $G_{2}(\lambda)$ равномерно ограничены. Третье слагаемое перепишем в виде

$$
\left[A^{1 / 2} \widetilde{L}_{0}^{-1}(\lambda) A^{1 / 2}\right]\left[A^{-1 / 2} B_{R}^{1 / 2}\right]\left[B_{R}^{-1 / 2} \widetilde{B} B_{R}^{-1 / 2}\right]\left[\lambda B_{R}^{1 / 2} \widetilde{L}^{-1}(\lambda)\right] .
$$

Все сомножители в этом произведении являются ограниченными функциями в $\mathbb{C}^{+}$. Действительно, первый сомножитель совпадает с $G_{1}(\lambda)$, а ограниченность четвертого доказана в лемме 10 . Так как $B$ - секториальный оператор, то, согласно второй теореме о представлении, $B \subset B_{R}^{1 / 2}(I+i S) B_{R}^{1 / 2}$, где $\|S\| \leqslant \operatorname{tg} \varphi$, а потому третий сомножитель ограничен в $\mathscr{H}$. Из оценки $(4)$ имеем при $x \in \mathscr{H}_{1}$

$$
\left\|B_{R}^{1 / 2} x\right\|^{2}=\operatorname{Re}(\widetilde{B} x, x) \leqslant \text { const }\left\|A^{1 / 2} x\right\|^{2},
$$

откуда следует, что оператор $B_{R}^{1 / 2} A^{-1 / 2}$ ограничен. Но тогда ограничен оператор $A^{-1 / 2} B_{R}^{1 / 2} \subset\left(B_{R}^{1 / 2} A^{-1 / 2}\right)^{*}$. Тем самым доказана ограниченность функции $F_{2}(\lambda)$.

Заметим, что $(\widetilde{B})^{*}$ совпадает с расширением $B^{*}$ как оператора из $\mathscr{H}_{1}$ в $\mathscr{H}_{-1}$. Поэтому, повторяя рассуждения леммы 10, получим равномерную ограниченность функции $A^{1 / 2}\left(\widetilde{L}^{*}(\bar{\lambda})\right)^{-1}$, где $\widetilde{L}^{*}(\lambda)=\lambda^{2}+\lambda \widetilde{B}^{*}+A$. Но тогда функция $F_{3}(\lambda)$ также равномерно ограничена в $\mathbb{C}^{+}$. Лемма доказана.

ЛЕмма 12. Функиия $F_{1}(\lambda)$ равномерно ограничена в $\mathbb{C}^{+}$в норме пространства $\mathscr{H}$. 
ДокАЗАтЕльство. Умножим равенство (11) на $A^{1 / 2}$ слева и на $B_{R}^{1 / 2}$ справа. Получим

$$
\begin{aligned}
A^{1 / 2} \widetilde{L}^{-1}(\lambda) B_{R}^{1 / 2}=A^{1 / 2} \widetilde{L}_{0}^{-1}(\lambda) B_{R}^{1 / 2}+A^{1 / 2} \widetilde{L}_{0}^{-1}(\lambda) \widetilde{A}^{1 / 2} \lambda \widetilde{L}^{-1}(\lambda) B_{R}^{1 / 2} \\
+A^{1 / 2} \widetilde{L}_{0}^{-1}(\lambda) \widetilde{B} \lambda \widetilde{L}^{-1}(\lambda) B_{R}^{1 / 2} .
\end{aligned}
$$

Все три слагаемых в правой части являются равномерно ограниченными операторами в $\mathbb{C}^{+}$. Действительно, $A^{1 / 2} \widetilde{L}_{0}^{-1}(\lambda) B_{R}^{1 / 2}=G_{1}(\lambda)\left(A^{-1 / 2} B_{R}^{1 / 2}\right)$; поэтому равномерная ограниченность первого слагаемого следует из ограниченности оператора $A^{-1 / 2} B_{R}^{1 / 2}$ и равномерной ограниченности оператор-функции $G_{1}(\lambda)$. Далее, второе слагаемое равно $G_{1}(\lambda) F_{2}^{*}(\bar{\lambda})$, а потому также равномерно ограничено в $\mathbb{C}^{+}$. Наконец, третье слагаемое можно переписать в виде

$$
\left[A^{1 / 2} \widetilde{L}_{0}^{-1}(\lambda) A^{1 / 2}\right]\left[A^{-1 / 2} B_{R}^{1 / 2}\right]\left[B_{R}^{-1 / 2} \widetilde{B} B_{R}^{-1 / 2}\right]\left[\lambda B_{R}^{1 / 2} \widetilde{L}^{-1}(\lambda) B_{R}^{1 / 2}\right] .
$$

Ранее было показано, что первые три сомножителя в этом произведении равномерно ограничены, а четвертый сомножитель обладает тем же свойством в силу леммы 10. Следовательно, функция $A^{1 / 2} \widetilde{L}^{-1}(\lambda) B_{R}^{1 / 2}$, а потому и $B_{R}^{1 / 2} \widetilde{L}^{-1}(\lambda) A^{1 / 2}$ равномерно ограничены в $\mathbb{C}^{+}$.

Умножив равенство (11) на $A^{1 / 2}$ с обеих сторон и воспользовавшись теми же аргументами, что и ранее, получим, что функция $\lambda^{-1} A^{1 / 2} \widetilde{L}^{-1}(\lambda) \widetilde{A}^{1 / 2}$ также равномерно ограничена в $\mathbb{C}^{+}$. Лемма доказана.

Утверждение теоремы 1 теперь следует из лемм 9-12.

\section{ЛитЕРАТУРА}

1. Thompson W. (Lord Kelvin), Tait P. Treatise on Natural Philosophy, Part 1. Cambridge University Press, 1869.

2. Четаев Н. Г. Устойчивость движения. Наука, М., 1990.

3. Chen G., Russel D. L. A mathematical model for linear elastic systems with structural damping. Quart. Appl. Math., 39, 433-454 (1982).

4. Chen S., Triggiani R. Proof of extensions of two conjectures on structural damping for elastic systems. Pacific J. Math., 136, No. 1, 15-55 (1989).

5. Chen S., Triggiani $R$. Gevrey class semigroups arising from elastic systems with gentle dissipation: the case $0<\alpha<1 / 2$. Proc. Amer. Math. Soc., 110, No. 2, 401-415 (1990).

6. Huang $F$. On the mathematical model for linear elastic systems with analytic damping. SIAM J. Control Optim., 26, No. 3, 714-724 (1988).

7. Huang F. Some problems for linear elastic systems with damping. Acta Math. Sci., 10, No. 3, 316-326 (1990).

8. Гринив P. О., Шкаликов A. А. Операторные модели в теории упругости и гидромеханике и ассоциированные с ними аналитические полугруппы. Вестник МГУ, сер. матем., мех., №5, 5-14 (1999).

9. Гринив P. О., Шкаликов А. А. Экспоненциальная устойчивость полугрупп, связанных с некоторыми операторными моделями в механике. Матем. заметки, 73, №5, 657-664 (2003).

10. Veselić K. Energy decay of damped systems. Представлена в Z. Angew. Math. Mech.

11. Kaто T. Теория возмущений линейных операторов. Мир, М., 1972.

12. Любич Ю. И. Классическое и локальное преобразование Лапласа в абстрактной задаче Коши. УМН, 21(129), №3, 3-51 (1966). 
13. Pazy A. Semigroups of Linear Operators and Applications to Partial Differential Equations. Applied Mathematical Sciences, Vol. 44, Springer-Verlag, New York-Berlin, 1983.

14. Engel K. J., Nagel R. One-Parameter Semigroups for Linear Evolution Equations. Springer-Verlag, Berlin-Heildelberg-New York, 2000.

15. Paidoussis M. P., Issid N. T. Dynamic stability of pipes conveying fluid. J. Sound Vibration, 33, 267-294 (1974).

16. Зефиров В. Н., Колесов В. В., Милославский А. И. Исследование собственных частот прямолинейного трубопровода. Изв. АН СССР, серия мех. тв. тела, №1, 179-188 (1985).

17. Datko R. Extending a theorem of A. M. Liapunov to Hilbert space. J. Math. Anal. Appl., 32, 610-616 (1970).

18. van Casteren J. A. Operators similar to unitary or selfadjoint ones. Pacific J. Math., 104, No. 1, 241-255 (1983).

19. Набоко C. Н. Об условиях подобия унитарным и самосопряженным операторам. Функц. анализ и его прил., 18, вып. 1, 16-27 (1984).

20. Маламуд M. M. Критерий подобия замкнутого оператора самосопряженному. Укр. матем. ж., 37, №1, 49-56 (1985).

21. Rolewicz $S$. On uniform $N$-equistability. J. Math. Anal. Appl., 115, 431-441 (1986).

22. Littman $W$. A generalization of a theorem of Datko and Pazy. In: Lecture Notes in Control and Inform. Sci., Vol. 130, Springer-Verlag, Berlin-New York, 1989, pp. 318323.

23. Гомилко А. М. Об условиях на производящий оператор равномерно ограниченной $C_{0}$-полугруппы операторов. Функц. анализ и его прил., 33, вып. 4, 66-69 (1999).

24. Маламуд M. M. О подобии треугольного оператора диагональному. Зап. научн. семин. ПОМИ, 270, 201-241 (2000).

25. Gearhart L. Spectral theory for contraction semigroups on Hilbert spaces. Trans. Amer. Math. Soc., 236, 385-394 (1978).

26. Shkalikov A. A. Operator pencils arising in elasticity and hydrodynamics: the instability index formula. In: Operator Theory: Adv. and Appl., Vol. 87, Birkhäuser, 1996, pp. 358-385.

Институт прикладных проблем механики и математики, Львов, Украина

Поступило в редакцию email:rhryniv@iapmm.lviv.ua 10 марта 2004 г.

Московский государственный университет, механико-математический факультет email: ashkalikov@yahoo.com 